\section{Qualidade e eqüidade na assistência à gestante: um estudo de base populacional no Sul do Brasil}

\author{
Quality and equity in prenatal care: a population- \\ based study in Southern Brazil
}

\begin{abstract}
This population-based study aimed to evaluate prenatal coverage according to family income in a municipality (county) in Southern Brazil. Data were collected using a questionnaire with mothers in the first 24 hours postpartum. Prenatal coverage, first prenatal consultation in the first trimester, six or more consultations, breast and colposcopic examination, routine prenatal laboratory tests according to the protocol of the Program for Humanization of Prenatal and Delivery Care (PHPN), and prenatal ultrasound increased proportionally with family income, and all were more frequent in women from the highest income quartile $(p<0.001)$. Despite these results, the prevalence of low birth weight did not show a statistically significant difference between the quartiles. The local health service appeared not to be very effective, since only $26.8 \%$ of the women were classified as having received adequate prenatal care according to the PHPN criteria, and care was unequal, since quality of care for women in the lowest income quartile was inferior to that of women in the highest quartile.
\end{abstract}

Prenatal Care; Quality of Health Care; Equity in Health
Carla Vitola Gonçalves 1

Juraci Almeida Cesar 1

Raul A. Mendoza-Sassi 1

\section{Introdução}

A assistência pré-natal compreende um conjunto de procedimentos que objetiva prevenir, diagnosticar e tratar eventos indesejáveis à gestação, ao parto e ao recém-nascido. Sua ausência e/ou deficiência está relacionada a maiores índices de morbimortalidade materna e perinatal 1,2,3,4,5. No Brasil, apesar da ampliação na cobertura pré-natal, chegando a $95 \%$ em algumas regiões e do aumento no número de consultas (1,2 consulta/parto em 1995, para 5,1 consultas/parto em 2003), ainda se observa que a redução dos riscos à gravidez com conseqüente melhora dos indicadores de saúde materna e fetal está longe do idealizado 2,5,6 (Departamento de Informática do SUS. Censos demográficos e contagem populacional, para os anos intercensitários, estimativas preliminares dos totais populacionais, estratificadas por idade e sexo. http://www.da tasus.gov.br).

Em 2005, o Ministério da Saúde instituiu a Política Nacional de Atenção Obstétrica e Neonatal 7 , considerando a necessidade de ampliar os esforços para alcançar as metas estabelecidas pelo Pacto Nacional para Redução da Mortalidade Materna e Neonatal, lançado pelo Ministério da Saúde no ano de 2004 8. Neste mesmo ano, o Ministério da Saúde publicou um manual técnico sobre atenção qualificada e humanizada no pré-natal e puerpério, que recomenda iniciar o acompanhamento no primeiro trimestre de gra- 
videz e realizar no mínimo seis consultas durante todo o pré-natal ${ }^{6}$. Além disso, recomenda realizar exame clínico das mamas, colpocitológico de colo uterino, verificação da pressão arterial e avaliação da altura uterina e do peso. Em relação aos exames laboratoriais, os de sangue, urina, anti-HIV e sorologia para sífilis deveriam ser realizados no primeiro trimestre e repetidos até a 30a semana de gestação. A partir da 20a semana, dever-se-ia iniciar suplementação com sulfato ferroso e apresentar imunização completa para o tétano a menos de cinco anos.

Análise dos dados disponíveis no Brasil mostra que apesar da alta cobertura pré-natal encontrada, há comprometimento na qualidade da atenção prestada ${ }^{9}$. Segundo o Ministério da Saúde, somente pequena parcela das gestantes inscritas nos programas de pré-natal consegue realizar o elenco mínimo de ações preconizadas 6,10. Essa situação traduz a inefetividade dos serviços de saúde quanto a garantir atendimento adequado não apenas em relação ao número de consultas, mas também ao conteúdo do atendimento oferecido 4,11. Além disso, alguns estudos revelam que mulheres com baixa renda, idade inferior a vinte anos e falta de acesso a plano privado de saúde, apresentam maior probabilidade de receber assistência pré-natal de qualidade inferior $4,11,12$. Talvez isso se explique em virtude de estas mulheres apresentarem menor poder de pressão sobre os serviços de saúde na reivindicação de atendimento de qualidade 11 . Esses achados confirmam a lei dos cuidados inversos, formulada por Hart 13, em 1971, indicando que as pessoas com maior necessidade de cuidados de saúde são as que menos os recebem, e que, quando os recebem, são de baixa qualidade. Portanto, estes serviços, ao invés de corrigir, acentuam as diferenças porque discriminam as mulheres mais pobres.

O processo de avaliação qualitativa da assistência pré-natal apresenta algumas dificuldades, pois trata da observação do resultado em face dos serviços recebidos ou que deveriam ser recebidos 4,14. Em função dessas dificuldades, há poucos estudos visando à avaliação da qualidade dos serviços a despeito de sua importante contribuição para redução da morbimortalidade materna e perinatal ${ }^{15}$. A própria literatura especializada tem privilegiado a análise das características e dos resultados da assistência, pelo número de consultas pré-natais e tipo de parto, relegando a um segundo plano o estudo da qualidade do conteúdo das consultas, embora este tipo de avaliação retrate com maior fidelidade o processo do atendimento 7,11,16,17,18.

O presente estudo teve por objetivo avaliar a cobertura da assistência pré-natal, a realização da rotina básica preconizada pelo Progra- ma de Humanização no Pré-natal e Nascimento (PHPN) e as características do parto conforme a renda familiar entre gestantes do Município de Rio Grande, Rio Grande do Sul, Brasil, que tiveram parto em 2007.

\section{Metodologia}

Este estudo foi realizado no Município do Rio Grande, que possui cerca de 200 mil habitantes e é localizado na planície costeira sul do Estado do Rio Grande do Sul. Sua economia é bastante diversificada e o seu produto interno bruto (PIB) per capita/ano é de aproximadamente $\mathrm{R} \$ 19.000,00$. No quesito desenvolvimento, ocupa a 29a posição dentre os 453 municípios do Rio Grande do Sul, com um o Indice de Desenvolvimento Humano (IDH) de 0,793. A sua rede de saúde é constituída por 32 unidades básicas, dois hospitais gerais e cinco ambulatórios. Pelo menos $99 \%$ dos partos ocorrem em hospitais. Apesar de o coeficiente de mortalidade infantil ter declinando nos últimos nos, chegando a 16/mil, ainda é superior à média do estado, com cerca de 13/mil (Departamento de Informática do SUS. Censos demográficos e contagem populacional, para os anos intercensitários, estimativas preliminares dos totais populacionais, estratificadas por idade e sexo. http://www.datasus.gov.br).

O delineamento utilizado para o estudo foi do tipo transversal de base populacional. Foram incluídos na pesquisa todos os nascimentos ocorridos nas duas únicas maternidades do município (Santa Casa de Misericórdia de Rio Grande e Hospital Universitário da Universidade Federal do Rio Grande - FURG) entre 1o de janeiro e 31 de dezembro de 2007. Estes recém-nascidos deveriam ter peso igual ou superior a $500 \mathrm{~g}$ ou vinte semanas ou mais de idade gestacional, e suas mães deveriam residir na área urbana ou rural do município e ter realizado pelo menos uma consulta de pré-natal.

Dez entrevistadoras foram treinadas para atuar neste estudo. Dentre elas, oito eram acadêmicas do curso de Medicina da FURG e as outras eram graduadas em Serviço Social. O treinamento consistiu na leitura do questionário e do manual de instruções, na sua aplicação entre duplas e perante o grupo de entrevistadores. O estudo piloto foi realizado nas duas maternidades da cidade com o objetivo de testar o questionário a ser utilizado bem como avaliar o tempo necessário à realização da entrevista.

Os entrevistadores visitavam diariamente as duas maternidades da cidade, que distam não mais que 100 metros uma da outra. Todas as informações do estudo foram coletadas por 
um questionário único pré-codificado, com as mães sendo entrevistadas na maternidade em até 24 horas após o parto. O questionário investigou características demográficas; nível sócioeconômico; história reprodutiva; assistência recebida durante a gestação e o parto; conhecimento sobre amamentação e imunização. Entre as variáveis referentes à qualidade do pré-natal, temos o índice de Kessner, que foi considerado adequado quando as gestantes iniciavam o prénatal no primeiro trimestre e realizavam seis ou mais consultas. Quanto à avaliação da rotina pré-natal, ela considerada adequada quando a gestante realizou seis ou mais consultas de prénatal, iniciou o pré-natal no primeiro trimestre, foi pesada, teve a pressão arterial verificada, a medida uterina aferida, as mamas palpadas, o exame ginecológico realizado e foi submetida a pelo menos dois exames de sangue, urina, HIV e sífilis, recebeu suplementação com sulfato ferroso e tinha imunização completa contra o tétano neonatal 19,20.

A identificação das gestantes era feita por informação obtida diretamente junto à gestante e confirmada por consulta ao livro de registro de nascimento de cada maternidade. Ao encontrar alguma parturiente residente no Município de Rio Grande, explicava-se a ela sobre os objetivos do estudo e a convidava para dele participar. Em havendo concordância, um termo de consentimento era assinado pela gestante autorizando a realização da entrevista. Ao final de cada dia de trabalho, o entrevistador codificava os questionários por ele aplicados e, no dia seguinte, os entregava na sede do estudo onde os questionários eram revisados e entregues à digitação.

A digitação dos questionários era duplamente realizada por digitadores independentes, em ordem inversa ao do primeiro. Ao término da digitação de cada lote, realizava-se comparação das digitações. A entrada de dados foi realizada com o Epi Info 6.04 (Centers for Disease Control and Prevention, Atlanta, Estados Unidos). Em seguida, realizou-se análise de consistência, que consistiu na criação e categorização de variáveis e verificação de freqüências realizadas por intermédio do programa Stata versão 9.2 (Stata Corp., College Station, Estados Unidos). Para as comparações entre proporções, utilizou-se teste do qui-quadrado com correção de Yates. O nível de significância foi de $95 \%$. O protocolo de pesquisa foi submetido e aprovado pelo Comitê de Ética em Pesquisa na Área da Saúde da FURG. Além disso, garantiu-se a confidencialidade dos dados, a participação voluntária e a possibilidade de deixar o estudo a qualquer momento, sem necessidade de justificativa.

\section{Resultados}

Dentre as 2.557 puérperas entrevistadas, 2.449 (96\%) haviam realizado pelo menos uma consulta de pré-natal. Na distribuição da renda familiar, verificou-se que $25 \%$ das puérperas encontravam-se no menor quartil (de $\mathrm{R} \$ 0,00$ até $\mathrm{R} \$ 450,00$ ), $27 \%$ no segundo quartil (de $\mathrm{R} \$ 451,00$ até $\mathrm{R} \$ 900,00), 23 \%$ no terceiro quartil (de $\mathrm{R} \$ 901,00$ até $\mathrm{R} \$ 1.350,00$ ), e $25 \%$ no quarto quartil ( $\mathrm{R} \$ 1351,00$ ou mais).

A média de idade das entrevistadas foi de 25,6 anos. No entanto, $20,2 \%$ das puérperas apresentaram idade igual a 19 anos ou menor; no primeiro quartil a taxa encontrada foi de $32,2 \%$ e no quarto quartil apenas $9,5 \%$. Ainda em relação à distribuição da amostra, 70,2\% das pacientes relataram-se brancas, $83,5 \%$ apresentavam companheiro fixo, $53,1 \%$ possuíam nove anos ou mais de escolaridade, $42,8 \%$ tinham de 2 a 3 gestações e $80,9 \%$ referiram nunca ter tido aborto (Tabela 1).

Na Tabela 2, observa-se que a cobertura prénatal aumenta progressivamente com a melhoria da renda familiar, passando de $90,6 \%$ no primeiro quartil para 98,9\% no quarto quartil (p < 0,001). Quanto ao ingresso no pré-natal 73,5\% das gestantes o fizeram ainda no primeiro trimestre, sendo, em média, com 13 semanas de idade gestacional. Gestantes pertencentes ao quartil de maior renda iniciaram mais freqüentemente as consultas no primeiro trimestre $(p<0,001)$. A média de consultas realizadas por gestante foi de 7,$4 ; 75,3 \%$ das entrevistadas tiveram seis ou mais consultas. Entretanto, no grupo de menor renda, a média de consultas foi de 5,9 e apenas $63 \%$ das pacientes realizaram seis consultas ou mais. Já as gestantes do quarto quartil realizaram, em média, 9,4 consultas: 90,2\% consultaram seis ou mais vezes $(p<0,001)$. Ainda nesta mesma tabela foi avaliada a realização dos exames clínicos do pré-natal em relação ao quartil de renda. Verifica-se que a pressão arterial foi medida em 98\% das gestantes não apresentando diferença estatística entre os quartis, assim como a medição da altura uterina. No entanto, a pesagem foi menor no grupo de maior renda, apresentando $\mathrm{p}=0,04$. Em relação ao exame ginecológico, ele foi pouco realizado, pois apenas $47,1 \%$ das puérperas tiveram suas mamas examinadas e $58,7 \%$ realizaram exame especular. Tais variáveis também apresentaram associação significativa entre maior quartil de renda e sua maior realização $(\mathrm{p}<$ 0,001). Quanto à execução de pelo menos dois exames de laboratório da rotina pré-natal mínima do PHPN e a realização da ultra-sonografia, constata-se que os procedimentos foram mais realizados entre gestantes do quartil de maior 
Distribuição da amostra conforme o quartil de renda em estudo de base populacional, no Sul do Brasil, 2007.

\begin{tabular}{|c|c|c|c|c|c|c|}
\hline \multirow[t]{2}{*}{ Variável } & \multicolumn{4}{|c|}{ Quartil de renda * (\%) } & \multirow[t]{2}{*}{ Total (\%) } & \multirow[t]{2}{*}{ Valor de $p$} \\
\hline & $\begin{array}{l}\text { Primeiro } \\
\text { (menor) }\end{array}$ & Segundo & Terceiro & $\begin{array}{l}\text { Quarto } \\
\text { (maior) }\end{array}$ & & \\
\hline Idade (anos completos) & & & & & & $<0,001$ \\
\hline $15-19$ & 32,2 & 21,5 & 17,2 & 9,5 & 20,2 & \\
\hline $20-24$ & 31,5 & 31,1 & 27,4 & 21,2 & 27,9 & \\
\hline $25-29$ & 19,2 & 24,7 & 25,4 & 29,6 & 24,7 & \\
\hline 30 ou mais & 17,1 & 22,7 & 30,0 & 39,7 & 27,2 & \\
\hline Média (desvio-padrão) & $23,5(6,3)$ & $25(6,3)$ & $26(6,4)$ & $28(6,4)$ & $25,6(6,5)$ & $<0,001$ \\
\hline Cor da pele & & & & & & $<0,001$ \\
\hline Branca & 61,1 & 70,4 & 71,8 & 77,8 & 70,2 & \\
\hline Parda & 23,1 & 17,3 & 18,2 & 13,4 & 18,0 & \\
\hline Negra & 15,8 & 12,3 & 10,0 & 8,8 & 11,8 & \\
\hline Viviam com companheiro & 74,8 & 83,6 & 86,3 & 89,2 & 83,5 & $<0,001$ \\
\hline Escolaridade (anos) & & & & & & $<0,001$ \\
\hline $0-4$ & 20,7 & 14,4 & 6,7 & 4,4 & 11,6 & \\
\hline $5-8$ & 52,0 & 43,1 & 31,9 & 14,4 & 35,3 & \\
\hline 9 ou mais & 27,3 & 42,5 & 61,4 & 81,2 & 53,1 & \\
\hline Média (desvio-padrão) & $6,7(2,7)$ & $7,7(2,9)$ & $9(2,4)$ & $10,2(2,2)$ & $8,4(2,9)$ & $<0,001$ \\
\hline Gestações & & & & & & $<0,001$ \\
\hline Primigesta & 39,5 & 38,3 & 41,8 & 43,0 & 40,7 & \\
\hline $2-3$ & 38,9 & 42,4 & 43,6 & 46,5 & 42,8 & \\
\hline 4 ou mais & 21,6 & 19,3 & 14,6 & 10,5 & 16,5 & \\
\hline Média (desvio-padrão) & $2,3(1,7)$ & $2,3(1,7)$ & $2,1(1,3)$ & $2(1,2)$ & $2,2(1,5)$ & 0,50 \\
\hline Aborto & & & & & & 0,50 \\
\hline $\operatorname{Sim}$ & 17,9 & 19,8 & 18,9 & 19,8 & 19,1 & \\
\hline Não & 82,1 & 80,2 & 81,1 & 80,2 & 80,9 & \\
\hline
\end{tabular}

* Quartil de renda: primeiro quartil (de $R \$ 0,00$ até $R \$ 450,00)$, segundo quartil (de $R \$ 451,00$ até $R \$ 900,00$ ), terceiro quartil (de $R \$ 901,00$ até $R \$ 1.350,00)$, quarto quartil ( $R \$ 1.351,00$ ou mais).

renda $(\mathrm{p}<0,001)$. Porém, apenas $25,3 \%$ das gestantes realizaram os dois exames sorológicos para sífilis, preconizados pelo PHPN - este se mostrou uma constante entre os diferentes quartis, não havendo diferença estatística significante. Em relação à suplementação com sulfato ferroso, ela foi significativamente mais prescrita para o grupo de menor renda. Quando avaliamos o prénatal de acordo com o índice de Kessner, verificase que $64,8 \%$ das gestantes realizaram pré-natal considerado adequado. Ao se associar ao índice de Kessner os exames clínicos, laboratoriais, suplementação com ferro e imunização completa contra o tétano neonatal, apenas $26,8 \%$ dos prénatais foram classificados como adequados. Este índice foi significativamente maior no grupo de maior renda $(p=0,01)$.
A prevalência de realização de cesariana foi significativamente maior entre aquelas gestantes do quarto quartil, sendo de $71,2 \%$ neste quartil contra $40,2 \%$ no primeiro quartil de renda. Quanto maior a sua renda, maior a chance de a parturiente e de o recém-nascido serem atendidos por um profissional médico no momento do parto $(\mathrm{p}<0,001)$. Ainda que a prevalência de baixo peso ao nascer tenha sido de $10,7 \%$ no primeiro quartil de renda contra $6,2 \%$ no melhor quartil, esta diferença não foi estatisticamente significativa. Praticamente 8 em cada 10 partos foram realizados pelo Sistema Único de Saúde (SUS). Observou-se ainda que quanto melhor o quartil de renda, maior a freqüência de partos pagos por meio de convênios ou particulares (Tabela 3 ). 
Assistência ao pré-natal conforme o quartil de renda em estudo de base populacional, no Sul do Brasil, 2007.

\begin{tabular}{|c|c|c|c|c|c|c|}
\hline \multirow[t]{2}{*}{ Variável } & \multicolumn{4}{|c|}{ Quartil de renda * $(\%)$} & \multirow[t]{2}{*}{ Total (\%) } & \multirow[t]{2}{*}{ Valor de $p$} \\
\hline & $\begin{array}{l}\text { Primeiro } \\
\text { (menor) }\end{array}$ & Segundo & Terceiro & $\begin{array}{l}\text { Quarto } \\
\text { (maior) }\end{array}$ & & \\
\hline Fez pré-natal & 90,6 & 96,6 & 97,6 & 98,9 & 95,8 & $<0,001$ \\
\hline Iniciaram as consultas de pré-natal no primeiro trimestre & 63,9 & 69,0 & 76,6 & 85,0 & 73,5 & $<0,001$ \\
\hline Número de consultas realizadas & & & & & & $<0,001$ \\
\hline 6 ou mais & 63,0 & 69,7 & 78,9 & 90,2 & 75,3 & \\
\hline Média (desvio-padrão) & $5,9(3,4)$ & $6,9(3,5)$ & $7,7(3,6)$ & $9,4(3,6)$ & $7,4(3,7)$ & $<0,001$ \\
\hline \multicolumn{7}{|l|}{ Exames clínicos realizados } \\
\hline Pressão arterial & 97,4 & 98,0 & 97,4 & 99,2 & 98,0 & 0,06 \\
\hline Pesagem & 98,7 & 98,6 & 97,7 & 97,4 & 98,1 & 0,04 \\
\hline Altura uterina & 98,9 & 99,2 & 98,2 & 98,4 & 98,7 & 0,22 \\
\hline Palpação das mamas & 36,3 & 42,2 & 48,9 & 61,4 & 47,1 & $<0,001$ \\
\hline Exame ginecológico & 49,8 & 53,5 & 61,6 & 70,4 & 58,7 & $<0,001$ \\
\hline Citopatológico do colo uterino & 49,4 & 59,6 & 66,8 & 73,2 & 62,1 & $<0,001$ \\
\hline \multicolumn{7}{|l|}{$\begin{array}{l}\text { Foram submetidas a pelo menos dois dos seguintes } \\
\text { exames }\end{array}$} \\
\hline Sangue & 77,7 & 79,1 & 82,3 & 88,7 & 81,9 & $<0,001$ \\
\hline Urina & 69,6 & 70,1 & 73,5 & 82,7 & 73,9 & $<0,001$ \\
\hline HIV & 66,3 & 68,7 & 70,7 & 75,8 & 70,4 & $<0,001$ \\
\hline Sífilis & 25,4 & 24,2 & 26,3 & 25,3 & 25,3 & $<0,03$ \\
\hline Ultra-sonografia pélvica & 50,1 & 64,4 & 78,9 & 89,5 & 70,5 & $<0,001$ \\
\hline Uso de sulfato ferro durante a gravidez & 68,1 & 62,4 & 57,7 & 58,2 & 61,7 & $<0,001$ \\
\hline Imunização completa contra o tétano neonatal & 78,5 & 79,5 & 78,9 & 73,6 & 78,3 & $<0,001$ \\
\hline \multicolumn{7}{|l|}{ Realizaram pré-natal segundo Kessner } \\
\hline Adequado ** & 50,8 & 58,7 & 69,1 & 81,4 & 64,8 & $<0,001$ \\
\hline Rotina adequada $\star \star \star$ & 25,1 & 23,9 & 27,8 & 30,7 & 26,8 & 0,01 \\
\hline
\end{tabular}

* Quartil de renda: primeiro quartil (de $R \$ 0,00$ até $R \$ 450,00)$, segundo quartil (de $R \$ 451,00$ até $R \$ 900,00)$, terceiro quartil (de $R \$ 901,00$ até $R \$ 1.350,00)$, quarto quartil ( $\mathrm{R} \$ 1.351,00$ ou mais);

** Gestantes que realizaram seis ou mais consultas de pré-natal e iniciaram o pré-natal no primeiro trimestre;

*** Gestantes que realizaram seis ou mais consultas de pré-natal, iniciaram o pré-natal no primeiro trimestre, foram pesadas, tiveram a pressão arterial verificada, a medida uterina aferida, as mamas palpadas, o exame ginecológico realizado e foram submetidas a pelo menos dois exames de sangue, urina, HIV e sífilis, receberam suplementação com sulfato ferroso e tinham imunização completa contra o tétano neonatal.

\section{Discussão}

Uma limitação deste estudo diz respeito à validação de alguns dados colhidos por entrevistas, particularmente aqueles que dependem da memória e da boa vontade da informante. Há, neste ponto, a possibilidade de viés recordatório, visto que algumas mães podem ter esquecido ou omitido alguns dados durante a entrevista. Isso poderia ter sido minimizado pela pesquisa no cartão da gestante e no prontuário médico. Contudo, vários estudos mostram a baixa qualidade de preenchimento dos dados pelos profissionais de saúde, nestes dois documentos 4,16,17,18. Apesar das limitações, os resultados sugerem a viabilidade da metodologia utilizada já que todos os estudos que se utilizam deste método são igualmente afetados pelo erro recordatório.

Este estudo mostrou que no grupo de puérperas do primeiro quartil, $32,2 \%$ apresentavam idade igual ou menor a 19 anos, enquanto no quarto quartil apenas 9,5\% das puérperas tinham esta idade. Um resultado semelhante foi encontrado em Campinas, São Paulo, Brasil, onde a proporção de gestantes adolescentes foi três vezes maior entre as mulheres de menor renda 12 . 
Assistência ao parto conforme o quartil de renda em estudo de base populacional, no Sul do Brasil, 2007.

\begin{tabular}{|c|c|c|c|c|c|c|}
\hline \multirow[t]{2}{*}{ Variável } & \multicolumn{4}{|c|}{ Quartil de renda * (\%) } & \multirow[t]{2}{*}{ Total (\%) } & \multirow[t]{2}{*}{ Valor de $p$} \\
\hline & $\begin{array}{l}\text { Primeiro } \\
\text { (menor) }\end{array}$ & Segundo & Terceiro & $\begin{array}{l}\text { Quarto } \\
\text { (maior) }\end{array}$ & & \\
\hline Tipo de parto & & & & & & $<0,001$ \\
\hline Normal & 59,8 & 51,7 & 46,5 & 28,8 & 46,8 & \\
\hline Cesariana & 40,2 & 48,3 & 53,5 & 71,2 & 53,2 & \\
\hline Parto realizado por médico & 79,7 & 86,7 & 88,1 & 93,0 & 86,6 & $<0,001$ \\
\hline $\begin{array}{l}\text { O recém-nascido foi atendido por pediatra na sala } \\
\text { de parto }\end{array}$ & 81,8 & 84,8 & 89,5 & 92,6 & 87,1 & $<0,001$ \\
\hline Prevalência de baixo peso ao nascer $(<2.500 \mathrm{~g})$ & 10,7 & 9,2 & 9,3 & 6,2 & 8,9 & 0,23 \\
\hline Tipo de assistência & & & & & & $<0,001$ \\
\hline Público (SUS) & 97,2 & 90,6 & 80,4 & 44,0 & 78,2 & \\
\hline Privado (Convênio/particular) & 2,8 & 9,3 & 19,6 & 56,0 & 21,8 & \\
\hline
\end{tabular}

SUS: Sistema Único de Saúde.

* Quartil de renda: primeiro quartil (de $R \$ 0,00$ até $R \$ 450,00$ ), segundo quartil (de $R \$ 451,00$ até $R \$ 900,00$ ), terceiro quartil (de $R \$ 901,00$ até $R \$ 1.350,00$ ), quarto quartil (R\$1.351,00 ou mais).

Outros estudos também têm constatado que a proporção de adolescentes gestantes aumenta de forma inversamente proporcional à renda familiar 21,22.

A média da cobertura de pré-natal no $\mathrm{Mu}$ nicípio de Rio Grande é alta (96\%), e a grande maioria das gestantes $(73,5 \%)$ inicia as consultas ainda no primeiro trimestre, realizando em média 7,4 consultas. Todavia, quando são associados a estes parâmetros e à execução dos exames de laboratório da rotina pré-natal mínima do PHPN, os exames clínicos gineco-obstétricos, a suplementação com ferro e a imunização completa contra o tétano neonatal, apenas $26,8 \%$ dos pré-natais foram classificados como "adequado". Além disso, a qualidade da atenção pré-natal foi influenciada pelo quartil de renda da gestante, mostrando que as pacientes de menor renda tiveram um atendimento de menor qualidade.

Outros estudos realizados no Brasil corroboram com os resultados do presente trabalho. Em auditoria realizada em 653 prontuários das gestantes atendidas no pré-natal do Amparo Maternal (São Paulo), sob responsabilidade de Escola de Enfermagem da Universidade de São Paulo, 38,4\% das gestantes tinham o pré-natal classificado como adequado em relação ao índice de Kessner. No entanto, quando se incluíam a este índice os exames laboratoriais da rotina do PHPN, nenhuma paciente teve seu acompanhamento pré-natal classificado como adequado 4 .

Em Juiz de Fora, Minas Gerais, a cobertura pré-natal encontrada foi de 99\%, com média de
6,4 consultas. Entretanto, apenas 27,6\% dos prénatais foram considerados adequados em relação ao inicio do acompanhamento no primeiro trimestre e ter realizado seis ou mais consultas. Quando foram incluídos na avaliação os exames laboratoriais básicos, apenas 1,9\% permaneceu como adequado, e por fim, somando-se os procedimentos clínico-obstétricos, somente $1,1 \%$ foi considerado como adequado 18 . Tal realidade também foi observada em Caxias do Sul, Rio Grande do Sul, onde a cobertura pré-natal encontrada foi de $95,4 \%$, mas somente $44,3 \%$ das pacientes submeteram-se a todos os exames complementares preconizados pelo PHPN; e mesmo entre as gestantes que relataram seis ou mais consultas de pré-natal, $57,6 \%$ não haviam sido submetidas a todos os exames recomendados. Com base nos critérios estabelecidos pelo PHPN, apenas 35,2\% das gestantes incluídas no estudo de Caxias do Sul tiveram seu pré-natal classificados como adequado 10 .

Ao se analisar os procedimentos de pré-natal, tendo como parâmetro as normas técnicas do PHPN, verifica-se freqüência elevada da aferição da pressão arterial, do peso materno e da medida da altura uterina em todos os estratos sociais. Porém, a palpação das mamas e o exame ginecológico foram realizados em apenas $47,1 \%$ e 58,7\%, respectivamente. E no grupo do primeiro quartil, apenas $36,3 \%$ das gestantes tiveram suas mamas examinadas e 49,8\% realizaram exame ginecológico. Em estudo recente realizado na cidade de Rio Grande, a cobertura pré-natal encontrada 
foi de $96 \%$, com média de 7,3 consultas. Apesar disso, apenas $59,1 \%$ das pacientes apresentavam exame colpocitológico coletado a menos de 36 meses e o exame clínico das mamas havia sido realizado em $40,2 \%$ das gestantes. Este estudo também evidenciou que as gestantes com menor escolaridade, menor renda familiar e que realizaram o pré-natal nos postos de saúde do município foram as menos examinadas, mostra claramente a diferença na qualidade do atendimento prestado a estas gestantes 23,24.

Avaliação da qualidade do pré-natal realizado em Criciúma, Santa Catarina, revelou elevada realização da medida da altura uterina e da pressão arterial em torno de $98 \% 25$. Apesar disso, os procedimentos que necessitavam de maior envolvimento médico, entre eles o exame ginecológico especular e a palpação da mamas, foram realizados com menor freqüência 50,9\% e 50,6\%, respectivamente. Também foi evidenciado que as gestantes com maior renda tinham 1,5 vez mais chances de serem submetidas ao exame especular e 1,9 vez mais suas mamas examinadas, que o grupo de pacientes com menor renda. Os autores concluem que tais procedimentos dependem do médico e exige maior tempo de consulta. Este tempo não foi dedicado às mais pobres, talvez porque elas tendam a um menor poder de pressão sobre os serviços de saúde na reivindicação de atendimento de qualidade. Estes dados corroboram teses incômodas sobre o tema relacionado à exclusão social.

Quando avaliamos a rotina laboratorial mínima preconizada pelo PHPN, verificamos que nenhum dos exames foi realizado duas vezes em todas as pacientes e que as gestantes do menor quartil foram as menos submetidas aos exames de sangue e urina. Entre os exames avaliados, o exame da sífilis foi o que mostrou pior taxa de realização no pré-natal de Rio Grande, com média de 25,3\%. Em relação à realização de dois VDRL no pré-natal, outros estudos também verificaram a baixa freqüência deste exame, sendo de $0,2 \%$ em São Paulo, 11,1\% em Juiz de Fora 4,18. Em um país como o Brasil, que apresenta uma prevalência de $1,6 \%$ de parturientes com sífilis e uma incidência de 2,1 casos de sífilis congênita por mil nascidos vivos em 2006, é preciso conscientizar os médicos pré-natalistas da importância de solicitar o exame de VDRL 26,27,28.

Na avaliação da assistência ao parto e ao recém-nascido, destaca-se a alta taxa de cesárea em toda a amostra estudada $(53,2 \%)$, mas isso não é de estranhar, pois as informações do Ministério da Saúde apontam para um aumento progressivo do parto cesárea ao longo dos últimos anos. Estudos de coorte realizados em Pelotas, Rio Grande do Sul, em 1982 e 2004, que incluí- ram todas as crianças nascidas na área urbana, encontraram um aumento da taxa de cesarianas de $27,7 \%$ para $45,2 \% 22$. Quanto à elevada taxa encontrada no quarto quartil $(71,2 \%)$, ela é similar à divulgada pela Agencia Nacional de Saúde Suplementar (ANS) no setor privado, que em 2006 encontrou uma taxa de $80 \%$ 29. Em estudo realizado em Embu, Região Metropolitana de São Paulo, a freqüência de cesarianas foi de $32,5 \%$; e no primeiro estrato com melhores condições sócio-econômicas, a taxa foi de $38,5 \%$; já no quarto estrato com piores condições sócio-econômicas, a taxa foi de $27,2 \% 11$.

A mortalidade perinatal é considerada um indicador sensível da adequação da assistência obstétrica e neonatal e do impacto de programas de intervenção nesta área, pela relação estreita que guarda com a assistência prestada à gestante e ao recém-nascido 3,30,31,32. No Brasil, a taxa de mortalidade neonatal vem se mantendo estabilizada em níveis elevados, com pouca modificação do componente neonatal precoce, ocupando papel importante na taxa de mortalidade infantil no país ${ }^{8}$. Sabe-se que há uma concentração de óbitos na primeira semana e predominantemente nas primeiras horas de vida, estabelecendo-se uma relação cada vez mais estreita com a assistência de saúde dispensada à gestante $\mathrm{e}$ ao recém-nascido durante o período pré-parto, parto e atendimento imediato à criança no nascimento 3,33,34,35. Além disso, tanto a mortalidade pós-natal como a neonatal é mais elevada nos grupos sociais de baixa renda, refletindo a falta de acesso deste grupo à assistência qualificada $3,33,34,35,36$. Neste estudo observa-se claramente a relação entre a presença do médico e do pediatra no momento do parto e a renda familiar da parturiente. O grupo de maior renda foi atendido pelo obstetra em $93 \%$ das vezes e teve pediatra na sala de parto em $92,6 \%$ dos casos. No grupo de menor renda, a presença do médico obstetra e do pediatra em sala de parto foi de $79,7 \%$ e $81,8 \%$, respectivamente. Apesar de o grupo de maior renda ter tido melhor assistência médica no momento do parto, ela não alcançou a totalidade de pacientes nem mesmo neste estrato.

Quando ao baixo peso ao nascer, tem se verificado nos últimos anos um comportamento paradoxal. É percebida uma redução das diferenças entre as freqüências de baixo peso e uma estagnação deste indicador. As hipóteses são: maior número de intervenções por diagnóstico pré-natal de risco gestacional e o maior número de cesarianas agendadas. No presente estudo, a prevalência de baixo peso ao nascer foi de $8,9 \%$, apresentando taxa de $6,2 \%$ no quarto quartil e $10,7 \%$ no menor quartil, sem significância estatística entre os diferentes quartis de renda. Pucci- 
ni et al. 11 encontraram uma prevalência de baixo peso de $11,3 \%$ no primeiro estrato e $13,5 \%$ no último estrato, dados semelhantes aos encontrados neste estudo. Outros estudos também não têm encontrado associação entre baixo peso e renda familiar per capita 12,37 .

Estudos que avaliam a qualidade do pré-natal por meio de índices como Kessner, Adequacy of Prenatal Care Utilization (APNCU) e Graduated Index of Prenatal Care Utilization (GINDEX), em relação à prematuridade, baixo peso ao nascer e pequeno para idade gestacional, têm revelado resultados paradoxais. Segundo esses estudos, os índices de avaliação do pré-natal demonstram maior força de associação quando avaliamos cuidados pré-natais e a relação com neonatos pequenos para idade gestacional. Ao se analisar associações entre o cuidado pré-natal e baixo peso ao nascer ou prematuridade, deve-se ter cuidado com a escolha dos índices porque os resultados diferem substancialmente 38,39 . Tais fatos nos demonstram que em vez de avaliarmos baixo peso ao nascer, talvez devêssemos avaliar o peso em relação à idade gestacional, uma vez

\section{Resumo}

Estudo de base populacional com objetivo de avaliar a cobertura da assistência pré-natal, de acordo com a renda familiar, em município do Sul do Brasil. As informações foram coletadas por questionário, com as mães nas primeiras 24 horas após o parto. Observase que a cobertura pré-natal, o inicio das consultas no primeiro trimestre, a realização de seis consultas ou mais, a execução do exame das mamas e do exame especular, a realização dos exames de laboratório da rotina pré-natal do Programa de Humanização no Pré-natal e Nascimento (PHPN) e a realização da ultra-sonografia, aumentam com a melhoria da renda familiar, sendo mais realizados nas gestantes do quartil de maior renda $(p<0,001)$. Apesar destes resultados, a prevalência de baixo peso ao nascer não apresentou diferença estatística significante entre os diferentes quartis. O serviço local de saúde mostrou-se pouco efetivo porque apenas $26,8 \%$ dos pré-natais foram classificados como adequados, segundo parâmetros do PHPN, e desigual porque as pacientes do menor quartil de renda tiveram o acompanhamento da sua gestação com qualidade inferior à das pacientes do quarto quartil.

Cuidado Pré-Natal; Qualidade da Assistência à Saúde; Eqüidade em Saúde que trabalhamos com a hipótese do aumento de cesarianas agendadas principalmente no maior estrato.

Embora este estudo constate uma alta cobertura pré-natal na cidade do Rio Grande, o serviço local de saúde mostrou-se pouco efetivo e desigual em se tratando da realização da rotina pré-natal preconizada pelo Ministério da Saúde do Brasil. Pouco efetivo porque apenas $26,8 \%$ dos pré-natais foram classificados como adequados, segundo parâmetros do PHPN 6, e desigual porque as pacientes do menor quartil de renda tiveram o acompanhamento da sua gestação com qualidade inferior à das pacientes do quarto quartil. Sendo assim, os resultados aqui apresentados revelam a necessidade de melhorar a qualidade da atenção pré-natal oferecida no Município do Rio Grande. Ficou claro que é preciso motivar e capacitar os profissionais de saúde quanto à importância dos procedimentos da rotina pré-natal, especialmente o exame especular, a palpação das mamas e o exame de sífilis (VDRL).

\section{Colaboradores}

C.V. Gonçalves participou da análise estatística e elaboração do artigo. J. A. Cesar e R. A. Mendoza-Sassi colaboraram na elaboração do projeto de onde o estudo foi originado, supervisão da coleta e da entrada de dados, auxílio na análise de dados e na redação final do artigo.

\section{Agradecimentos}

A Pastoral de Criança pelo financiamento. 


\section{Referências}

1. Barbieri M, Silva AAM, Bettiol H. Risk factors for the increasing trend in low birth weight among live births born by vaginal delivery, Brazil. Rev Saúde Pública 2000; 34:596-602.

2. Barros FC, Victora CG, Barros AJ, Santos IS, Albernaz E, Matijasevich A, et al. The challenge of reducing neonatal mortality in middle-income countries: findings from three Brazilian birth cohorts in 1982, 1993, and 2004. Lancet 2005; 365:847-54.

3. Lansky S, França E, Leal MC. Mortalidade perinatal e evitabilidade: revisão de literatura. Rev Saúde Pública 2002; 36:759-72.

4. Koffman MD, Bonadio IC. Avaliação da atenção pré-natal em uma instituição filantrópica da cidade de São Paulo. Rev Bras Saúde Matern Infant 2005; 5:23-32.

5. Aerts DRG. Investigação dos óbitos perinatais e infantis: seu uso no planejamento de políticas públicas de saúde. J Pediatr (Rio J.) 1997; 73:364-6.

6. Ministério da Saúde. Pré-natal e puerpério: atenção qualificada e humanizada - manual técnico. Brasília: Ministério da Saúde; 2005.

7. Ministério da Saúde. Portaria no ${ }^{\circ}$ 1.067, de 4 de julho de 2005. Instituição da Política Nacional de Atenção Obstétrica e Neonatal. Diário Oficial da União 2005; 6 jul.

8. Ministério da Saúde. Portaria n ${ }^{\circ}$. 427/GM, de 18 de março de 2004. Pacto Nacional pela Redução da Mortalidade Materna e Neonatal. Diário Oficial da União 2004; 22 mar.

9. Santos SR, Cunha AJL, Gamba CM, Machado FG, Leal JMMF, Moreira NLM. Avaliação da assistência à saúde da mulher e da criança em localidade urbana da Região Sudeste do Brasil. Rev Saúde Pública 2000; 34:266-71.

10. Trevisan MR, Lorenzi DRS, Araújo NM, Ésber K. Perfil da assistência pré-natal entre usuárias do sistema único de saúde em Caxias do Sul. Rev Bras Ginecol Obstet 2002; 24:293-9.

11. Puccini RF, Pedroso GC, Silva EMK, Araújo NS, Silva NN. Eqüidade na atenção pré-natal e ao parto em área da Região Metropolitana de São Paulo, 1996. Cad Saúde Pública 2003; 19:35-45.

12. Almeida SDM, Barros MBA. Eqüidade e atenção à saúde da gestante em Campinas (SP), Brasil. Rev Panam Salud Pública 2005; 17:15-25.

13. Hart JT. The inverse care law. Lancet 1971; 1:405-12.

14. Adami NP, Maranhão AMSA. Qualidade dos serviços de saúde: conceitos e métodos avaliativos. Acta Paul Enferm 1995; 8:47-55.

15. Tanaka ACD'A, Siqueira AAF, Bafile PN. Situação de saúde materna e perinatal no Estado de São Paulo, Brasil. Rev Saúde Pública 1989; 23:67-75.

16. Dias-da-Costa JS, Madeira ACC, Luz RM, Britto MAP. Auditoria médica: programa de pré-natal em posto de saúde na Região Sul do Brasil. Rev Saúde Pública 2000; 34:329-36.

17. Silveira DS, Santos IS, Dias-da-Costa JS. Atenção pré-natal na rede básica: uma avaliação da estrutura e do processo. Cad Saúde Pública 2001; 17:131-9.
18. Coutinho T, Teixeira MTB, Dain S, Sayd JD, Coutinho LM. Adequação do processo de assistência pré-natal entre as usuárias do Sistema Único de Saúde em Juiz de Fora, MG. Rev Bras Gineco Obstet 2003; 25:717-24.

19. Kessner DM. Screening high-risk populations: a challenge to primary medical care. J Community Health 1976; 1:216-25.

20. Takeda SMP. Avaliação de unidade de atenção primária: modificação dos indicadores de saúde e qualidade da atenção [Dissertação de Mestrado]. Pelotas: Centro de Pesquisas Epidemiológicas, Universidade Federal de Pelotas; 1993.

21. Rebollo AG, Monteiro CM. Perinatal variables and health inequalities in a health care district in Cáceres, Spain. Gac Sanit 2000; 14:31-8.

22. Cesar JA, Matijasevich A, Santos IS, Barros AJD, Dias-da-Costa JS, Barros FC, et al. The use of maternal and child health services in three population-based cohorts in Southern Brazil, 1982-2004. Cad Saúde Pública 2008; 24 Suppl 3:S427-36.

23. Gonçalves CV, Dias-da-Costa JS, Duarte G, Marcolin AC, Garlet AF, Sakai AF, et al. Exame clínico das mamas em consultas de pré-natal: análise da cobertura e de fatores associados em município do Rio Grande do Sul Brasil. Cad Saúde Pública 2008; 24:1783-90.

24. Gonçalves CV, Dias-da-Costa JS, Duarte G, Marcolin AC, Lima LCV, Garlet G, et al. Avaliação da freqüência de realização do exame físico das mamas, da colpocitologia cervical e da ultrassonografia obstétrica durante a assistência pré-natal: uma inversão de valores. Rev Assoc Med Bras 2009; 55:290-5.

25. Neumann NA, Tanaka OY, Victora CG, Cesar JA. Qualidade e equidade da atenção ao pré-natal e ao parto em Criciúma, Santa Catarina, Sul do Brasil. Rev Bras Epidemiol 2003; 6:307-18.

26. Ministério da Saúde. Boletim Epidemiológico AIDS/DST 2007; IV(1).

27. Ministério da Saúde. Diretrizes para controle da sífilis congênita: manual de bolso. $2^{\text {a }}$ Ed. Brasília: Ministério da Saúde; 2006.

28. Ministério da Saúde. Protocolo para a prevenção de transmissão vertical de HIV e sífilis. Brasília: Ministério da Saúde; 2006.

29. Agência Nacional de Saúde Suplementar. Parto normal: movimento ANS em favor do parto normal e da redução das cesarianas desnecessárias no setor suplementar. http://www.ans.gov.br (acessado em 07/Mai/2008).

30. Liljestrand J. Reducing perinatal and maternal mortality in the world: the major challenges. $\mathrm{Br} \mathrm{J}$ Obstet Gynaecol 1999; 106:877-80.

31. Maranhão AGK, Joaquim MMC, Siu C. Mortalidade perinatal e neonatal no Brasil. Tema (proj. RADIS) 1999; 2:6-17.

32. Laurenti R, Buchalla CM. Indicadores da saúde materna e infantil e implicações da décima revisão da Classificação Internacional de Doenças. Rev Panam Salud Pública 1997; 1:18-22. 
33. Leal MC, Szwarcwald CL. Evolução da mortalidade neonatal no Estado do Rio de Janeiro, Brasil (19791993): análise por causa segundo grupo de idade e região de residência. Cad Saúde Pública 1996; 12:243-52.

34. Leal MC, Szwarcwald CL. Características da mortalidade neonatal no Estado do Rio de Janeiro na década de 80: uma visão espaço-temporal. Rev Saúde Pública 1997; 31:457-65.

35. Paulucci RS, Nascimento LFC. Mortalidade neonatal em Taubaté: um estudo caso-controle. Rev Paul Pediatr 2007; 25:358-63.

36. Leite AJM, Marcopito LF, Diniz RLP, Silva AVS, Souza LCB, Borges JC, et al. Mortes perinatais no município de Fortaleza, Ceará: o quanto é possível evitar? J Pediatr (Rio J.) 1997; 73:388-94.
37. Monteiro CA, Benício MHA, Ortiz LP. Tendência secular do peso ao nascer na cidade de São Paulo (1976-1998). Rev Saúde Pública 2000; 34:26-40.

38. VanderWeele TJ, Lantos JD, Siddique J, Lauderdale DS. A comparison of four prenatal care indices in birth outcome models: comparable results for predicting small-for-gestational-age outcome but different results for preterm birth or infant mortality. J Clin Epidemiol 2009; 62:438-45.

39. Heaman MI, Newburn-Cook CV, Green CG, Elliot LJ, Helewa ME. Inadequate prenatal care and its association with adverse pregnancy outcomes: a comparison of indices. BMC Pregnancy Childbirth 2008; 8:15.

Recebido em 31/Mar/2009

Versão final reapresentada em 26/Jun/2009

Aprovado em 01/Jul/2009 